\title{
LASERS AND HOLOGRAPHY
}


This page is intentionally left blank 


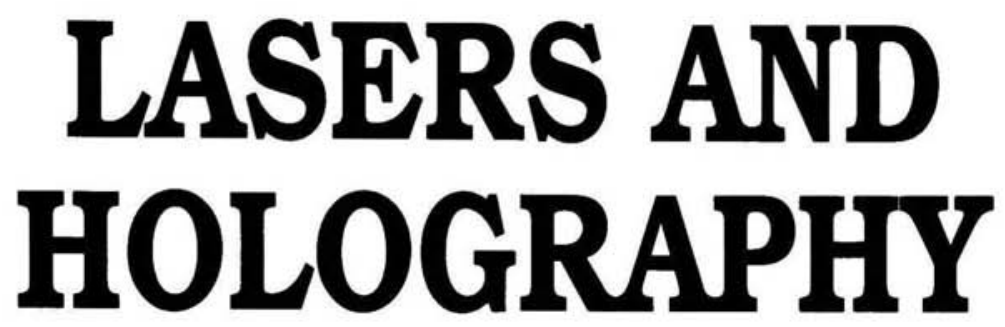

P C MEHTA

Instruments Research and Development Establishment Defence Research and Development Organisation Dehra Dun, India

\section{V RAMPAL}

Department of Science and Technology

New Delhi, India 
Published by

World Scientific Publishing Co. Pte. Ltd.

P O Box 128, Farrer Road, Singapore 9128

USA office: Suite 1B, 1060 Main Street, River Edge, NJ 07661

UK office: 73 Lynton Mead, Totteridge, London N20 8DH

\section{LASERS AND HOLOGRAPHY}

Copyright $\odot 1993$ by World Scientific Publishing Co. Pte. Ltd.

All rights reserved. This book, or parts thereof, may not be reproduced in any form or by any means, electronic or mechanical, including photocopying, recording or any information storage and retrieval system now known or to be invented, without written permission from the Publisher.

For photocopying of material in this volume, please pay a copying fee through the Copyright Clearance Center, Inc., 27 Congress Street, Salem, MA 01970, USA.

ISBN $981-02-1214-3$

Printed in Singapore. 


\title{
Dedicated to
}

\section{BHAPPO}

\author{
"The Candle burns \\ and brightens those \\ that cause it to burn"
}


This page is intentionally left blank 


\section{PREFACE}

The coming of lasers and their use for holography marked an important era in the advancement of physical sciences. This generated diverse fields of activity and gave new life to well researched areas like Raman spectroscopy. Holography provided an excited medium for three dimensional display. Lasers and holography continue to find new areas of applications. Their use in industry, medicine, communication and instrumentation for analysis and measurement are now well established. Individually, the two subjects, Lasers and Holography, have been treated at length in the literature and excellent reviews are available. The present book covers both the areas in a comprehensive manner. It is not always easy to get an overall view of a modern discipline in the presence of vast literature scattered in journals, books, reports and conference proceedings. Our effort of presenting both lasers and holography in a single volume may therefore appear rather ambitious but we hope it will achieve its purpose of providing the essentials of the topics and application update in a fairly reasonable extent.

The subject of holography is still evolving and a number of new applications such as computer interconnects and in medicine are emerging fast. It simply depends on ones imagination to exploit the unique features of holograms. A complete treatment of such applications and their thorough understanding therefore need further developments in architectures, techniques and materials.

The first three chapters discuss the physics and technology of lasers, while chapter 4 deals with the principles and techniques of holography. The chapters 5 to 9 describe the applications. The applications in the areas of medicine, information processing, nondestructive testing and interconnections have been included for their relevance in the context of recent developments.

In writing the book a large number of original publications have been used. However, the references cited do not represent the complete bibliography on the subject. Only those references are listed which have been used for writing the book. It is natural in such a book that many important publications may have been left unintentionally, for which 
the authors regret.

The book aims for a readership of those studying for a Master's degree in Applied Optics, Lasers and Holography and requiring an understanding of the generation and use of coherent optical radiation. The book will be useful to students, researchers and professionals actively involved in the development of lasers, holography and their applications. It is hoped that the students and researchers would find it useful and interesting.

one of the authors (PCM) is grateful to $\mathrm{Dr}$ O.P. Nijhawan, Director, IRDE, Dehra Dun for helpful suggestions. The authors are also thankful to $\mathrm{Mr}$. Devendra Mohan, $\mathrm{Mr}$. K.S.S. Rao, Mr. Chandra Bhan and Mr. Pritam Lal for providing some of the photographs used in the book. We take great pleasure in acknowledging the valuable help of Dr R.K. Tyagi and Mr. A.K. Musla for their critical reading of the manuscript. We wish to specially thank Mr. R.K. Kukreti for his patience and skill in typing. We are obliged to Mr. A.K. Musla for his painstaking efforts in type setting all the equations and tables, and formatting the manuscript in the computer for laser printing. Finally, we express our appreciation to the World Scientific Publishing Company for the high quality of publication.

The authors acknowledge the patience and moral support of their spouses, Dr. Mrs. P. Mehta (PCM) and Mrs. S. Rampal (VVR) during the writing of the book.

May 19, 1993. 


\section{CONTENTS}

PREFACE

vi i

1. OPTICAL RADIATION AND PHOTONS

1.1. Nature of light 1

1.1.1. Quantum description of radiation 4

1.1.2. Fluctuation properties

1.1.3. Power flow of electromagnetic radiation

1.2. Interaction of radiation with matter-

Emission and absorption of radiation

1.2.1. Transition probability

1.2.2. Emission and absorption of radiation by bound electrons

1.3. Spontaneous and stimulated radiation 20

1.4. Einstein coefficients 24

1.5. Optical gain 26

1.6. Gain saturation $\quad 29$

1.7. Optical resonators 31

1.7.1. Resonant modes of optical cavity 33

1.7.2. Theoretical methods for analyzing the 38

1.7.3. Gaussian beams 39

1.7.4. Design of open resonator cavity 45

1.7.5. ' $Q$ ' of optical resonator 52

1.7.6. Unstable resonators 54

1.8. Threshold condition for laser oscillation 57

1.9. Cavity coupling 59

1.10. Frequency of resonant modes 6

1.11. Frequency selection of laser oscillation 61

1.12. Transverse modes selection 62

1.13. Longitudinal mode selection 63

1.14. Mode competition $\quad 65$

1.15. Hole burning $\quad 66$

1.16. Mode pulling $\quad 67$

1.17. Frequency stability of laser output 72

1.18. Single mode operation of a laser 73

1.19. Coherence of laser radiation 74

1.19.1. Time coherence 75 
1.19.2. Spatial cohernerat

1.19.3. Time and space coherence

1.19.4. Transient coherence

1.19.5. Higher order coherence functions

1.19.6. Factors responsible for imparting coherence to laser radiation

1.20. Laser noise

1.21. General treatment of laser oscillation

2. SPATIAL, TEMPORAL AND SPECTRAL CHARACTERISTICS OF LASER

2.1. Introduction

2.2. Mode locking

2.3. Methods of mode locking

2.3.1. Mode locking with saturable absorberPassive mode locking

2.3.2. Active mode locking

2.3.3 Acousto-optic and electro-optic modulators as mode locking devices

2.3.4. Self mode locking

2.3.5. Experimental arrangement of an active model locked laser

2.3.6. Stabilization of mode locked lasers

2.4. Measurement on mode locked pulses

2.5. Generation and measurement of ultrashort pulses

2.5.1. Synchronous mode locking

2.5.2. Colliding pulse mode locking

2.5.3. Self phase modulation and pulse compression

2.5.4. Measurement of ultrafast pulses

2.6. Mode locking of transverse modes

2.7. Q-switching and cavity dumping

2.7.1. Cavity dumping

2.7.2. Q-switching

2.8. Relaxation oscillation

3. SPECIFIC LASER SYSTEMS

3.1. Solid state lasers

3.1.1. The Ruby laser

3.1.2. Neodymium lasers

3.1 .3 . Tunable solid state lasers

3.1.4. Sensitized solid state laser materials

3.1.5. Eye safe solid state lasers 
3.1.6. Diode laser pumping of solid state lasers

3.1.7. The slab lasers

3.2. Colour centre lasers

3.3. Semiconductor lasers

3.3.1. The $\mathrm{p}-\mathrm{n}$ junction laser diode 178

3.3.2. Heterojunction lasers 180

3.3.3. Recent advances

3.3.4. Quantum well lasers

3.3.5. Distributed feedback lasers

184

184

3.4. Dye lasers

188

193

3.4.1. The dye as laser medium

193

$3 \cdot 4.2$. Spectra of organic dyes

195

3.4.3. Requirements for starting oscillation

199

3.4.4. Cavity arrangements

3.4.5. Output characteristics

200

3.4.6. Specific purpose developments 205

3.5. Gas lasers

206

3.5.1. He-Ne laser

206

3.5.2. Argon ion laser

209

3.5.3. Carbon dioxide laser 210

3.5.4. TEA $\mathrm{CO}_{2}$ laser 212

3.5.5. Gas dynamic $\mathrm{CO}_{2}$ laser 215

3.6. Chemical lasers

218

3.6.1. HF/DF laser

219

3.7. Carbon monoxide (CO) laser

3.8. Excimer lasers
3.9. Nitrogen laser

221

224

3.10. Metal vapour lasers

226

3.10.1. He-cd laser

228

3.10 .2 . $\mathrm{Hg}-\mathrm{Br}$ laser

228

3.10.3. Copper vapour laser

229

3.10.4. Plasma recombination laser

231

234

3.11. Far infrared (FIR) lasers

238

3.12. Free electron laser (FEL) nonlinear processes

3.13.1. Second order effects in nonlinear crystals- Generation of second harmonics

3.13.2. Third order nonlinear processes in gaseous media- Generation of tunable UV and IR 
4. HOLOGRAPHY:PRINCIPLES AND TECHNIQUES

4.1. Introduction

4.2. Characteristics of a hologram

4.3. In-line Holography: Gabor holography

4.4. Off-axis Holography: Leith-Upatnieks holography

4.5. Holographic imaging equations

4.6. Image magnification

4.7. Hologram aberrations

4.8. Orthoscopic and pseudoscopic images

4.9. Classification of holograms

4.9.1. Amplitude and phase holograms

4.9.2. Classification based on hologram thickness

4.9.3. Classification based on direction of reconstructed image

4.9.4. Classification according to recording arrangement

4.10. Practical holography

4.10.1. Laser

4.10.2. Reference-to-object intensity ratio

4.10.3. Angle between reference and object beams

4.10.4. Polarization of light beams

4.10.5. Vibration isolation table

4.10.6. Optical components and mounts

$4 \cdot 10.7$. Hologram recording geometries

4.10.8. Hologram of a moving object

4.10.9. Efficiency of a hologram

4.10.10 Refractive index modulation

4.10.11. Signal-to-Noise Ratio (SNR)

4.11. Holographic recording materials

4.11.1. Modulation transfer function

4.11.2. Nonlinear recording

4.11.3. Silver halide emulsion

318

4.11.4. Hardened dichromated gelatin (DCG)

4.11.5. Photopolymers

4.11.6. Photoresists

4.11.7. Photothermoplastics

4.11.8. Photochromic materials

4.11.9. Photorefractive crystals (Electrooptic materials)

4.11.10. Summary of recording materials

4.11.11. Health hazards of hologram processing chemicals 
$4.12 .2 \cdot 360^{\circ}$ hologram

380

$4 \cdot 12.3$. Rainbow hologram

385

4.12.4. Holographic stereogram

398

4.12 .5 . Viewing zone

4.12 .6 . Change of size

402

4.12.7. Dispersion compensation

403

4.12 .8 . Sources for reconstruction

404

4.12.9. Hologram display systems

408

4.12.10. Holographic 3D printer

408

4.12.11. Holographic television

412

4.12 .12 . Holographic cinematography

414

414

4.13. Colour holography

416

4.13.1. Chromaticity diagram

4.13 .2 . Recording of colour holograms

416

4.13.3. Volume colour holograms

418

4.13.4. Recording geometry

418

4.13.5. Pseudocolouring

423

426

4.14. Special techniques

4.14.1. Local reference beam hologram

4.14.2. Multiple-exposure holography (Scanning object beam holography)

4.14.3. Multiplexed hologram

4.14.4. Multifaceted hologram

4.14.5. Pinhole hologram

439

4.14.6. Edge-lit hologram

443

4.15. Hologram replication

445

4.15.1. Optical interferometric techniques

445

4.15.2. Mechanical replication technique

(Embossed holograms)

449

4.16. Polarization holography

4.16.1. Depolarization effects

4.16.2. Polarization recording

4.17. Evanescent wave holography (Waveguide holography)

4.17.1. Holographic lithography

5. HOLOGRAPHIC INTERFEROMETRY

5.1. Introduction

5.2. Double-exposure holographic interferometry

5.3. Single-exposure real-time holographic interferometry

5.4. Time-average holographic interferometry
5.5. Stroboscopic holographic interferometry

5.6. Temporally modulated holography 
5.7. Fringe linearization holographic interferometry

5.8. Desensitized holographic interferometry 489

5.9. Digital holographic interferometry

5.10. Fringe localization

5.10.1. Pure translation

5.10.2. Pure rotation about an axis in the surface

5.11. Sandwich hologram interferometry

5.12. Applications

5.12.1. Holographic nondestructive testing (HNDT)

5.13. Holographic contouring

5.13.1. Two wavelength holographic contouring

5.13.2. Two refractive index holographic contouring

$5 \cdot 13 \cdot 3$. Contouring by change in the illuminating angle

5.14. Holographic interferometry with fibre optics

6. HOLOGRAPHIC OPTICAL ELEMENTS

6.1. What is a HOE?

6.2. Hologram of a point

6.3. Resolution of a HOE

6.4. Design aspects

6.5. Fabrication

6.6. Holographic gratings/mirrors

6.7. Applications of HOEs

6.7.1. Spectral filters

6.7.2. Applications in optical communication

6.7.3. HOEs in compact disks

6.7.4. Holographic laser beam attenuator

6.7.5. HOE based fibre optic gyroscope

6.7.6. Holographic scanner

6.7.7. Diffractive-refractive telescope

6.7.8. Applications in architecture

6.7.9. Beam combiners

6.7.10. Fingerprint sensor

6.7.11. HOEs in Art

7.1. Introduction

7.2. Optical interconnects

classification of holographic interconnects

7.4. HOE size

7.5. Desirable characteristics 